\title{
Thoracoscopic surgery for bronchobiliary fistula: a case report
}

\author{
Yen-Shou Kuo ${ }^{1}$, Shih-Chun Lee ${ }^{1}$, Hung Chang ${ }^{1}$, Chung-Bao Hsieh ${ }^{2}$ and Tsai-Wang Huang ${ }^{1 *}$
}

\begin{abstract}
The bronchobiliary fistula is an abnormal interconnection between the biliary tract and bronchial trees. It is rare but troublesome. The management of such fistula is a challenge. Surgical intervention is considered after failure of conservative treatment. Here we presented the successful video-assisted thoracoscopic surgery for secondary bronchobiliary fistula in 68-year-old hepatocellular carcinoma patient.
\end{abstract}

Keywords: Bilioptysis, Biliary fistula, Bronchial fistula, Thoracoscopic surgery

\section{Background}

A bronchobiliary fistula (BBF) is a rare condition that was first reported by Peacock in 1850 [1]. A BBF is an abnormal connection between the biliary tract and bronchial tree. An acquired BBF is generally regarded to be a consequence of a local infection, trauma, biliary tract obstruction, or neoplasm as most are related to hepatic trauma, subphrenic abscess/infection and hepatocellular carcinoma. The presence of bilioptysis and repeated infection could make the diagnosis. However, managing this fistula is a challenge and is often associated with high morbidity and mortality rates. After conducting a literature search, there is surprising very little information regarding thoracoscopic management for an acquired BBF. Here we present our experience with a thoracoscopic intervention for an acquired BBF that, to the best of our knowledge, has not been previously reported.

\section{Case presentation}

A 68-year-old Asian male, nonsmoker was referred to our ward with consolidation in the right lower lung (Figure 1) accompanied by green sputum and bitter saliva. He had been diagnosed with stage I non-B, non-C hepatocellular carcinoma of the liver in segment 4 , which was treated with laparotomy and central lobectomy 2 years ago before this admission. Six months later after operation, he underwent

\footnotetext{
* Correspondence: chi-wang@yahoo.com.tw

'Division of Thoracic Surgery, Department of Surgery, Tri-Service General Hospital, National Defense Medical Center, 325, Cheng-Kung Road 2nd section, Taipei 114, Taiwan

Full list of author information is available at the end of the article
}

percutaneous transhepatic cholangial drainage and endoscopic retrograde cholangiopancreatography (ERCP) with plastic stent implantation because of postoperative strictures of common bile duct. The stent was removed 3 months before the current presentation. At the current admission, physical examination revealed decreased breath sounds in the right lower lung field. Dyspnea was not observed. A reverse L-shaped scar measuring approximately $12 \mathrm{~cm}$ in length was noted on the right upper quadrant of the abdomen. There was no rebound tenderness or muscle guarding. The sputum was positive for bile. Chest computed tomography (CT) confirmed consolidation of the right lower lung (Figure 2A\&B). Flexible bronchoscopy revealed that the right lower bronchus was coated with bile-like material (Figure 3A\&B). These findings led to the suspicion of a BBF. The biochemical study of sputum was positive for bilirubin.

Under single-lung anesthesia ventilation, we performed a minimally invasive video-assisted thoracic surgery. Using a 10 - $\mathrm{mm}, 30$-degree thoracoscope, a $10 \mathrm{~mm}$ trocar was placed through the sixth intercostal space in the midaxillary line. A $2 \mathrm{~cm}$ incision was placed in the fourth intercostal space in the anterior axillary line. A ring forceps was used through this incision to displace the lung posteriorly and expose the adhesion site between the right lower lung lobe and the diaphragm (Figure 4A\&B). After adhesion lysis, disconnection between the lung and diaphragm was performed smoothly using an Endo-GIA.

The patient was discharged 5 days after surgery. There were no signs of bilioptysis with 1 year follow up. 


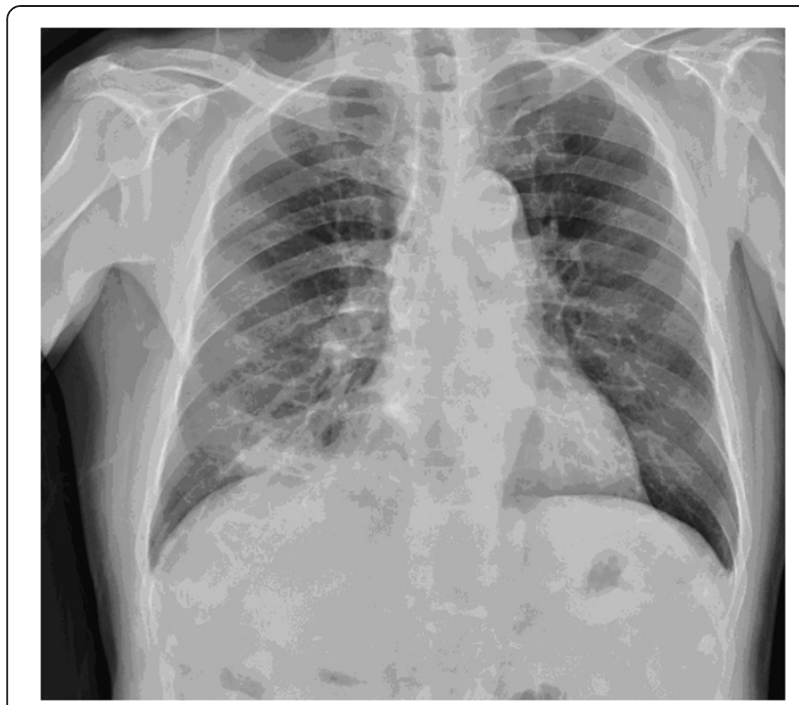

Figure 1 Shadows are noted in the lower right region of the lungs on a chest X-ray.

\section{Discussion}

Here we reported a case of an secondary BBF that was clinically suspected and confirmed by contrast-enhanced $\mathrm{CT}$ of the abdomen. The optimal treatment for BBF remains controversial. A number of reports have advocated nonsurgical or conservative therapy as an alternative in some cases. As shown by Yilmaz et al. [2], Singh et al. [3], and Ertugrul et al. [4], conservative therapy with biliary and abscess drainage can be effective for BBF associated with a liver abscess, liver hydatidosis after surgical cyst removal, or biliary tract obstruction due to stones or short strictures and for selected cases of post-traumatic BBF. For other cases, surgery is probably the best option.

Liao et al. [5] considered that open surgery should be the first choice when interventional techniques have failed or if a BBF has developed secondary to tumors, biliary obstruction, or trauma. The type of surgery depends on the primary tumor type, BBF location, and involvement of other structures. The following surgical procedures have been performed for a BBF: drainage of a right subphrenic or hepatic abscess, fistula closure, resection of hydatid cysts or a tumor, biliary drainage caused by a T-tube, and bilioenteric anastomoses. For patients with diaphragmatic, pleural, bronchial, or pulmonary damage, diaphragm closure, pleural drainage, decortication, or different pulmonary resections have been performed. There was no mention of thoracoscopic surgery in our review of the literature. In this presenting case, percutaneous hepatic drainage catheter injury or liver abscess is the possible etiology of BPF. It was difficult to differentiate. However, the stent could not resolve the patient's symptoms. Biloma persisted even with the stent . So we decided to perform VATS procedure for this patient.
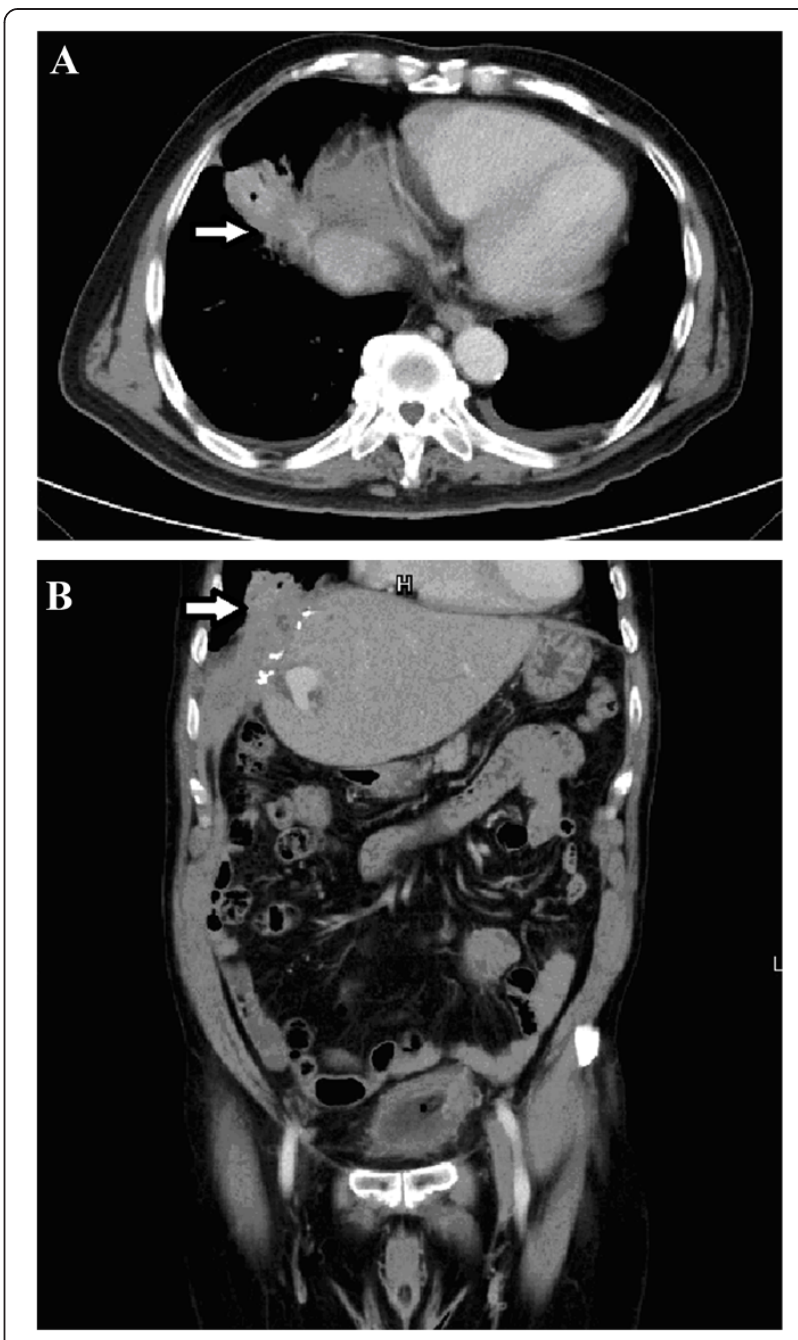

Figure 2 Computer tomography of abdomen. A: The axial view showed a lobulated heterogenous mass lesion in the right lower lobe. B: The coronal view showed a lobulated heterogeneous lesion in the surgical area over segment 4 of the liver which was adjacent to the diaphragm. Lobulated mass above the diaphragm was also noted.

We performed a minimally invasive video-assisted thoracoscopic resection of a BBF, following which the patient showed an amelioration of bilioptysis and was uneventfully discharged. Compared with previous case reports of surgical treatment by thoracotomy, video-assisted thoracoscopic surgery can be performed safely, with a shorter hospital stay and similar mortality and complication rates. Eryigit et al. [6] described three patients with BBF's that were treated by thoracotomy. The hospital stay for these patients was $>10$ days. Although the minimally invasive operation could result in less postoperative pain and shorter hospital stay, thoracoscopic surgery for a BBF is complexity and difficulty for surgeons. The repeated infection resulted in severe adhesion of lung. With the advanced surgical technique and equipment, the thoracoscopic procedure should be 

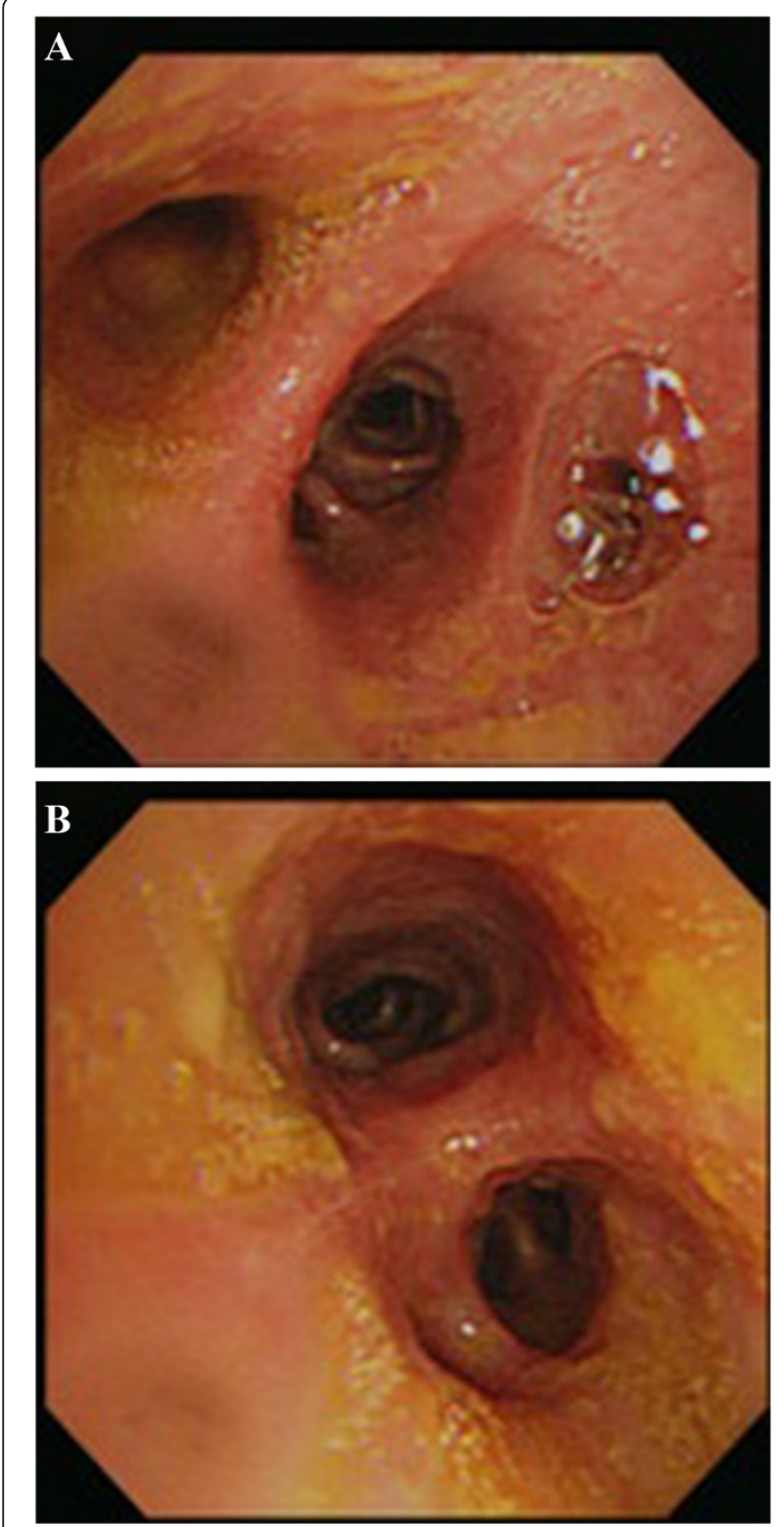

Figure 3 Flexible bronchoscopy. A: Yellowish material coating the right lower bronchus. B: Yellowish material coating the carina.

considered for treating a BBF. We presented successful management experience in BPF via VATS procedure. We thought that it may be possible, but not for all case. VATS management of BBF may be limited to early BBF.

\section{Conclusion}

In conclusion, the present case report suggests that minimally invasive, video-assisted thoracoscopic resection is a safe and effective means for treating a selective BBF.

\section{Consent}

Written informed consent was obtained from the patient for publication of this Case report and any accompanying
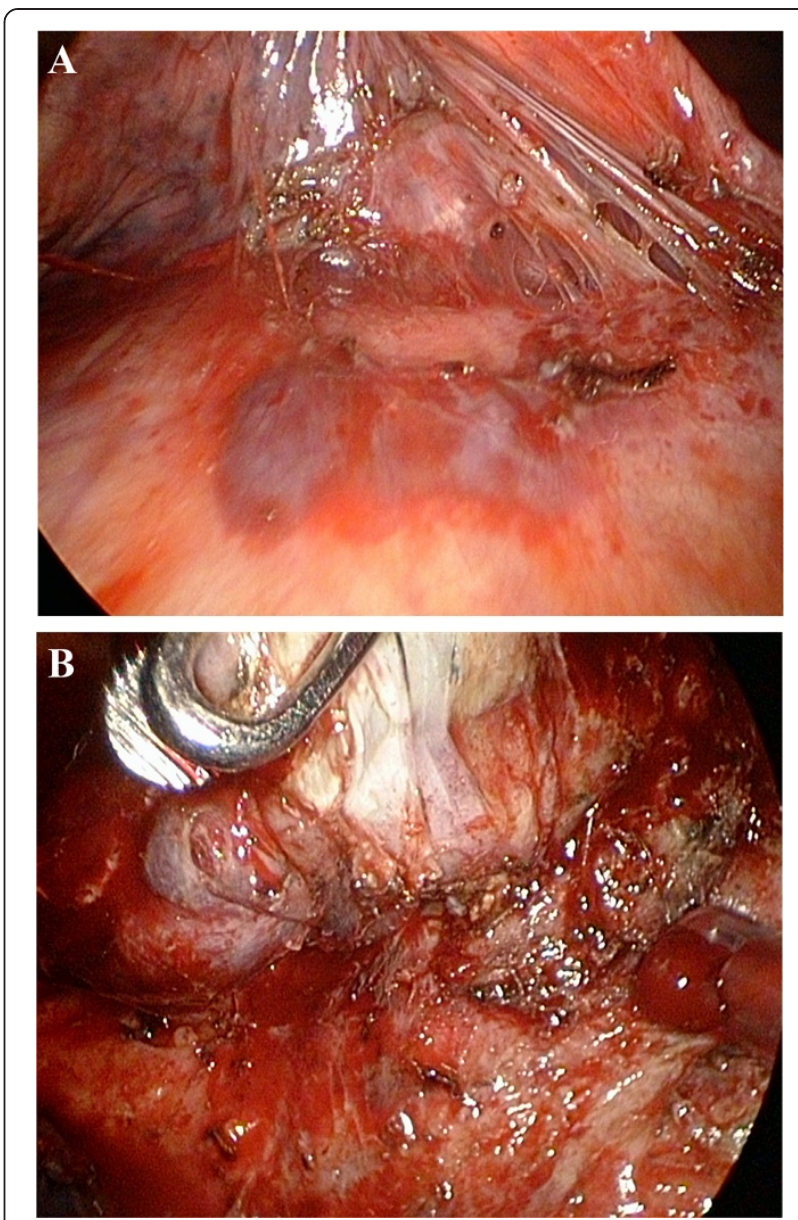

Figure 4 Thoracosopic finding. A: Severe adhesion between the diaphragm and lung parenchyma of the right lower lobe. B: Pneumolysis is performed and resection of the bronchobiliary fistula $(B B F)$ is achieved.

images. A copy of the written consent is available for review by the Editor-in-Chief of this journal.

\section{Abbreviations}

BBF: Bronchobiliary fistula; CT: Computed tomography.

\section{Competing interests}

There are no any financial competing interests (political, personal, religious, ideological, academic, intellectual, commercial or any other) to declare in relation to this manuscript.

\section{Authors' contributions}

YSK, TWH, HC and SCL carried out the VATS procedure in this case report; CBH carried out central lobectomy for the patient. YSK and TWH participated in the sequence alignment and drafted the manuscript. All authors read and approved the final manuscript.

\section{Authors' information}

Yen-Shou Kuo

Education

2004 2009 National Defense Medical Center

Department of Medicine

2000 2002 Taipei Municipal Jianguo High School

Working Experiences

08/2012 03/2014 Resident training, Tri-Service General Hospital. 


\section{Acknowledgements}

There were no other personnel that were involved in the acquisition of information or who contributed towards the paper that did not qualify for authorship.

\section{Author details}

${ }^{1}$ Division of Thoracic Surgery, Department of Surgery, Tri-Service General Hospital, National Defense Medical Center, 325, Cheng-Kung Road 2nd section, Taipei 114, Taiwan. ²Division of General Surgery, Tri-Service General Hospital, National Defense Medical Center, Taipei, Taiwan.

Received: 30 March 2014 Accepted: 4 August 2014

Published online: 18 September 2014

\section{References}

1. Peacock TB: Case in which hydatids were expectorated and one of suppuration of hydatid cyst of the liver communicating with the lungs. Edinburgh Med J 1850, 74:33-46.

2. Yilmaz U, Sahin B, Hilmioglu F, Tezel A, Boyacioglu S, Cumhur T: Endoscopic treatment of bronchobiliary fistula: report on 11 cases. Hepatogastroenterology 1996, 43:293-300.

3. Singh B, Moodley J, Sheik-Gafoor MH, Dhoma N, Reddi A: Conservative management of thoracobiliary fistula. Ann Thorac Surg 2002, 73:1088-1091.

4. Ertugrul I, Koklu S, Koksal AS, Coban S, Basar O, Ibis M, Sahin B: Treatment of bronchobiliary fistula due to an infected hydatid cyst by a nonsurgical approach. Dig Dis Sci 2004, 49:1505-1597.

5. Liao G-Q, Wang H, Zhu G-Y, Zhu K-B, Lv F-X, Tai S: Management of acquired bronchobiliary fistula: a systematic literature review of 68 cases published in 30 years. World J Gastroenterol 2011, 17:3842-3849.

6. Eryigit $\mathrm{H}$, Oztas $\mathrm{S}$, Urek S, Olgac G, Kurutepe M, Kutlu CA: Management of acquired bronchobiliary fistula: 3 case reports and a literature review. J Cardiothorac Surg 2007, 2:52.

doi:10.1186/s13019-014-0139-z

Cite this article as: Kuo et al.: Thoracoscopic surgery for bronchobiliary fistula: a case report. Journal of Cardiothoracic Surgery 2014 9:139.

\section{Submit your next manuscript to BioMed Central and take full advantage of:}

- Convenient online submission

- Thorough peer review

- No space constraints or color figure charges

- Immediate publication on acceptance

- Inclusion in PubMed, CAS, Scopus and Google Scholar

- Research which is freely available for redistribution 